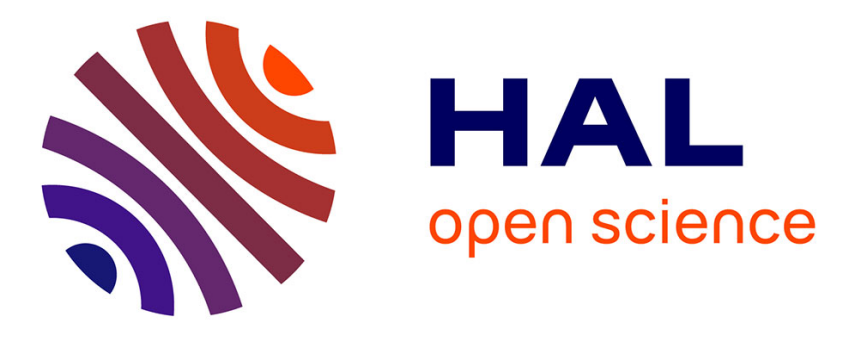

\title{
Toward Resilient and Efficient Maintenance Planning for Water Supply Networks
}

\author{
Marine Dubillard, Guillaume Martin, Matthieu Lauras, Xavier Lorca, Jean \\ Cantet
}

\section{- To cite this version:}

Marine Dubillard, Guillaume Martin, Matthieu Lauras, Xavier Lorca, Jean Cantet. Toward Resilient and Efficient Maintenance Planning for Water Supply Networks. 22nd Working Conference on Virtual Enterprises (PRO-VE 2021), Nov 2021, Saint-Etienne, France. pp.591-600, 10.1007/978-3-030-859695_55. emse-03346551

\section{HAL Id: emse-03346551 \\ https://hal-emse.ccsd.cnrs.fr/emse-03346551}

Submitted on 25 Nov 2021

HAL is a multi-disciplinary open access archive for the deposit and dissemination of scientific research documents, whether they are published or not. The documents may come from teaching and research institutions in France or abroad, or from public or private research centers.
L'archive ouverte pluridisciplinaire HAL, est destinée au dépôt et à la diffusion de documents scientifiques de niveau recherche, publiés ou non, émanant des établissements d'enseignement et de recherche français ou étrangers, des laboratoires publics ou privés. 
Dubillard M., Martin G., Lauras M., Lorca X., Cantet J. (2021) Toward Resilient and Efficient Maintenance Planning for Water Supply Networks. In: Camarinha-Matos L.M., Boucher X., Afsarmanesh H. (eds) Smart and Sustainable Collaborative Networks 4.0. PRO-VE 2021. IFIP Advances in Information and Communication Technology, vol 629. Springer, Cham. https://doi.org/10.1007/978-3-030-85969-5_55

\title{
Toward Resilient and Efficient Maintenance Planning for Water Supply Networks
}

\author{
Marine Dubillard, Guillaume Martin, Matthieu Lauras, \\ Xavier Lorca and Jean Cantet \\ University of Toulouse - Mines Albi, Industrial Engineering Department, Albi, France, ${ }^{2}$ \\ Veolia Eau France \\ \{marine.dubillard, guillaume.martin, matthieu.lauras, xavier.lorca\}@mines-albi.fr, \\ jean.cantet@veolia.com
}

\begin{abstract}
Every day, the water supply network evolves to fix or prevent leaks or contaminated drinking water, and hook-ups to buildings are set up or removed. Most technical interventions are planned in advance, but emergencies can abruptly disturb the planning. This work intends to propose a methodology to evaluate various planning strategies in order to maximize the resilience and efficiency of the water supply network scheduling. An industrial application is developed on a large French water management company. The baseline of this case is the existing planning process observed in the field. Then, based on a literature analysis, a set of alternative planning processes when removing some constraints. The proposed methodology allows to compare the existing solution to these alternatives. Finally, as the research is in its infancy, the paper develops avenues for future research through a specific research agenda.
\end{abstract}

Keywords: Water supply maintenance, Planning under uncertainty, Computer modelling, Robust planning.

\section{Introduction and Problem Statement}

Population increases in both developed and developing countries around the world constantly puts a greater stress on the needs for amenities. Tap water is one of the most vital of these. However, providing reliable access to it proves to be an increasingly complex task, both by creating new water networks and maintaining the older ones. This paper deals with the latter and especially with the routing of maintenance teams to planned or spontaneous tasks. Maintaining water supply networks calls for three types of actions: (i) first, the evolution of the network with new buildings to hook up, (ii) second, preventive maintenance such as sample collection and proactive leak search and (iii) reactive maintenance when failures occur in the network. While the first two types are predictable, the last is composed of emergencies, thus creating uncertainties in the planning. On top of these, real intervention times and real transportation times in a wide area add more sources of uncertainty to the problem. Both these types of uncertainty can be considered as a 
stochastic phenomenon, for data analysis and field expertise can provide the grounds for a probability distribution, rendering them predictable to a degree. In this study, we focus on the French regional scale. Consequently, we must consider both the effectiveness of the service provider, defined as the capability to deliver the expected service, and the efficiency, defined as the capability to limit the costs of operations, in the wake of the increasing concurrency of providers. In the meantime, the uncertainties in our problem warrant the construction of resilient and robust plans. We define a robust planning as a planning that is not significantly impacted by hazardous events. A resilient planning strategy is a strategy with a high capacity to go back to an acceptable state after having been impacted by a hazardous event. These definitions follow the works of [1]. A vast part of the literature analysing water network maintenance focuses on predictive algorithms that help to decide which canalizations are the most susceptible to fail and should be changed. For example, [2] compare various statistical models to evaluate the failure risk of pipes, using the history of pipe breaks and [3] review state-of-the-art models to use in order to predict the remaining useful life of individual pipes. But producing a proper estimate of the demand (maintenance operations) is only a part of the problem. The other part consists in using these estimates to feed optimized maintenance planning systems, able to better manage disruptions and cost-effectiveness. In such a context, our research question is the following: how to challenge and improve the maintenance planning strategies for water supply networks? To solve this issue, we share an applied methodology to improve this maintenance by considering the optimization of water production and distribution network maintenance planning in an uncertain environment. We start by reviewing the literature on dynamic planning problems. Section 3 then details our methodology to quantitatively compare different planning alternatives and dynamic routing approaches, while looking for resilient, efficient and robust options. Section 4 elaborates the first steps of this applied to a French regional case, along with specificities of their processes, and we conclude with avenues for future research.

\section{Smart and Collaborative Dynamic Routing Problems}

At its core, our maintenance planning problem has two components. It is a capacitated vehicle routing problem (CVRP) with water provider workers having to optimize their maintenance planning. It must also deal with planning uncertainty, rendering it dynamic, with emergency repairs appearing during the day.

Solving the capacitated vehicle routing problem. CVRP problems were first defined in [4] with the intention of finding optimal routes for several fuel vehicles to refill multiple stations. The capacity aspect of the problem is often represented as variable demands of customers, but it may also view task and travel time as consuming the vehicle capacity. The static version of the problem is most often posed as a set of customers with coordinates and varied demands, along with a number of available vehicles. Over the years, several varieties of the CVRP have been formulated: including time windows to fill customer requests (VRPTW in [5]), taking profit into account and allowing customers to be left out of the routes (CVRPP in [6]) or even adding pickup and delivery aspects in between customers (VRPPD in [7]). 
Interested readers may look for further details in the transformation of VRP problems to job shop scheduling problems in [8], a paper that can have implications in solving the problem in alternative ways. For a survey on heuristics and exact methods on how to solve VRPs and its variants, the reader may turn to [9]. An updated review, but only for exact methods, is also available in [10].

Dynamic workforce routing in uncertain environments. Dynamic vehicle routing problems (DVRP) are a variation on the classic vehicle routing problem where requests (usually in the form of new customers, but equivalent to emergencies in our case) pop up while vehicles are executing their route [11]. The 'dynamic' refers to the fact that these new requests (i) were not part of the starting set of requests and (ii) must be considered in the reconstruction of an updated route. Several methods are used in order to deal with these problems, as for example in the review of [11] and the use of periodic or constant re-optimization. The same authors, along with [12] for example, also consider tackling the problem by identifying the stochastic nature of customer appearance ahead of time. The literature mentions several ways to solve DVRP problems, such as [13] with a greedy random search procedure if the problem is viewed with the scheduling paradigm. Other approaches are available in a series of works from W. Kool and his co-authors, represented in [14]. In this recent paper, they train a reinforcement learning agent to dynamically adapt vehicle routes, thus leveraging the power of deep neural networks. Other meaningful resolution methods are found in [15] and the coupling of simulation software to metaheuristics. We must also mention recent advances in [16] and [17] using multiple stages algorithms implying a clustering of clients to visit for the DVRP. Several use cases were recently documented with the sharp increase of highly connected supply chains with contributions like [18] who consider dynamic optimization of supply chains. Other hyper connected network cases exist with the works of [19] and smart transportation management systems. The different cases of DVRP give rise to various types of uncertainties in the planning like in [20] or [21]. At that point, literature on the DVRP shows many alternatives to both model and solve different variants of the problem. In our case of water supply maintenance, we aim to evaluate if, and to what extent, the existing approaches fit our needs. Consequently, we wish to bridge the gap between statistical methods for predictive maintenance in water networks and dynamic routing of teams in a structured design of experiments. In parallel, by measuring performance against efficiency, resilience and robustness criteria, we should be able to conclude on our problem. The next section details our proposed methodology to answer this question.

\section{Proposed Research Methodology}

Our methodology is summarized by Figure 1. The first step consists in gathering the maximum amount of information on the existing model: what are the roles of each stakeholder, what are their constraints, how do they cooperate in planning routes with or without emergencies. The end goal of this first step is three-fold: (i) establish the meaningful rules and mechanisms governing the problem at hand, while getting rid of 
noise in order to build the simulation with the correct degree of precision, (ii) describe the static and dynamic planning methods already used in our use case, that should act as a comparison baseline and (iii) use the data we collected to tune our simulation model to reality, a step also called model calibration. The choice of using (discrete) simulation is motivated by its ability to model the necessary degrees of complexity [22]. Model calibration is an essential part of using simulations for decision-making (see also [22] for further details). The next step of the methodology is to build a list of alternative planning processes, either from the literature, or with new contributions. It must be noted that, for each alternative process, there may be variants due to hyperparameters or sets of constraints. These two aspects are taken into account by simulating different versions, much similar to a design of experiments. Lastly, each simulation run is evaluated against several performance criteria. We consider two sets of criteria: one coming from the use case, in order to link our results to business expectations, and one tied to resilience and efficiency aspects.

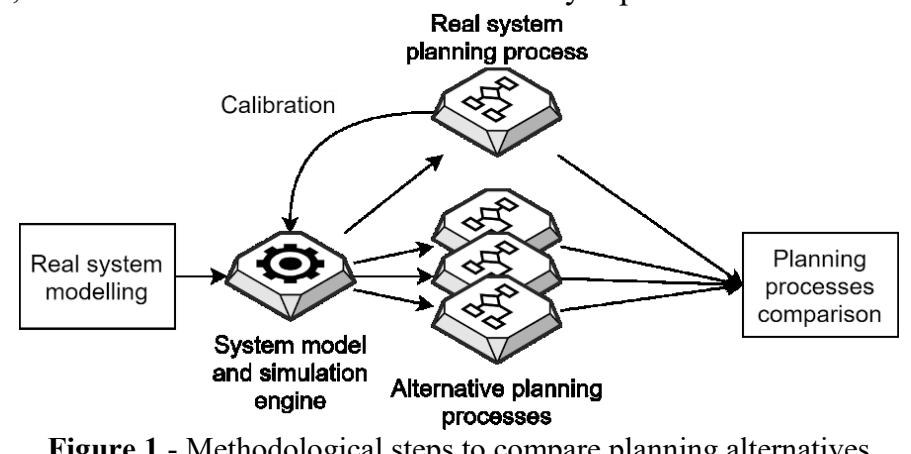

Figure 1 - Methodological steps to compare planning alternatives

Real system modelling. In order to establish a baseline, we started by conducting a series of interviews with the water maintenance actors. We asked two series of questions, one for each planning processing method: predictive scheduling (before the occurrence of disturbance during the execution) and reactive (after disturbance) scheduling. 5 people, each with a different role in the general planning and routing process were interviewed. Interviews contained semi-opened questions in order to guarantee a common base but still allow for a possibility to gather supplementary information [23]. In addition, we gathered historical planning data from planning tools over 2019 and 2020. Data covers planning and real execution. We also built a mathematical model of the problem, transforming data into tasks, and the information retrieved into resources and constraints, while staying as close as possible to the use case reality. Finally, we completed the model by concatenating the information into synthetic business processes (using the Business Process Model Notation).

Simulation engine building. Having only modelled the problem and the baseline process is not sufficient. More steps are needed in order to build the simulation engine. Each experiment we will carry will run on a parallel instance of the problem based on our data collection. As a result, we start by implementing a representative instance generator by fitting its probabilities of intervals and gravity to field data, as well as a failure generator simulating two kinds of uncertainties: the occurrence of an emergency intervention and the real processing time of each intervention. 
Real system planning process. Next the real system processes are implemented based on their previous modelling and can be run on the instance previously generated. Simulation calibration is then done by iterating over the model until behaviours match to a satisfying degree, by comparing output signals.

Alternative planning processes. Each alternative is either extracted from the literature or built by modifying the constraints of the baseline use case. More details in section 4 . We remind that each alternative must be fed with the same instance of the problem in order to give general conclusions.

Process performance comparison. Possible performance criteria to compare to outputs of real system and alternative processes include inverse schedule tardiness or inverse miss rate [24]. Other metrics follow a statistical approach, such as the standard deviations of routes, the entropy generated or the lateness likelihood of routes ([25], [26]). Feedback from the use case allows to add time spent on maintenance tasks over total working time, time and distance travelled and degree of unforeseen operations in a given time window. The result of these different steps is threefold as they will lead to (i) an exhaustive understanding of real field processes, (ii) a calibrated evaluation tool validated on a real basis, (iii) performance indicators reference values opening the way for improvement.

\section{Application Case : Veolia EAU Sud-Ouest}

Description of the ongoing application case specific problem. The application case is the water production and distribution network maintenance activity managed by the company Veolia Eau in 18 departments in the southwest of France. The planning problem consists in a set of interventions to execute, each having a precise location, a due date, a predictive processing time and a set of skills needed to intervene. The resources necessary are a set of agents, each having a specific set of skills, working hours and an agency location, which determines their position in the beginning and end of each work shift. The material resources issues are set aside as we consider that each agent has a car available at all times, and small intervention material available. The decision variables considered are the agent and the date associated with each intervention. The problem constraints are the following: for each intervention the due date has to be respected, and the agent in charge must have every skill needed. For each agent, the working hours have to be respected, and of course, time and space consistencies have to be preserved. This planning problem is rendered dynamic with the arrival of emergency interventions randomly popping and intervention times taking less or more time than expected. 


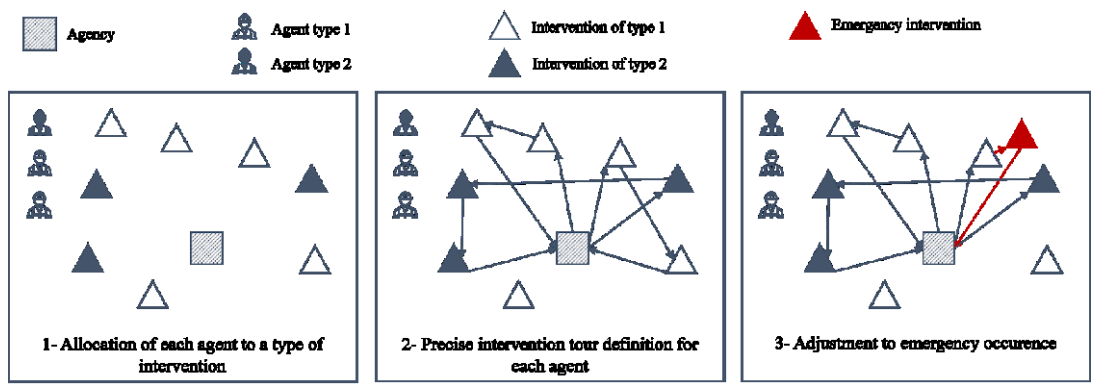

Figure 2. Planning decision steps in Veolia Eau

Planning processes in the ongoing application case. The complexity of this general problem is reduced with some additional organizational constraints specific to Veolia Eau: the planning processes are executed in small geographic areas, and each executing team composed of 5 to 10 agents has its own interventions set to handle. Moreover, the planning processes are executed each week with a weekly horizon, and each agent is allocated to a specific type of intervention every week. Finally, the interventions on emergencies are only added to an agent's schedule with no replanning process, and if an intervention lasts longer than expected, the remaining interventions in the schedule are just shifted in consequence. This organization makes the planning problem small enough to be handled by human intelligence and divides it into three subproblems depicted by Figure 2: (i) the allocation of each agent to a specific type of intervention, (ii) the intervention tour definition for each agent, consisting in selecting the interventions to execute and their order, (iii) the choice of an agent to handle an emergency occurring during the intervention tour.

All these steps are executed manually, with practice field intelligence. The allocation of agents to intervention types is made according to the general volume of each intervention type. The intervention tour is decided for each agent, one at a time, in the form of an ordered list of interventions to execute, with the objective of filling their schedules with the maximum number of interventions, while prioritizing timesensitive interventions. When emergencies occur during the execution of interventions, one agent is chosen according to a geographic criterion and interrupts his tour to intervene. When the emergency is fixed, he executes the other planned interventions in the same planned order, with the risk of not being able to execute the last interventions of the day.

The representation of these processes in a computer tool requires only simple algorithms and a CVRP resolution on a small number of interventions and one agent. The results are not expected to be consequently better than human scheduling and their quality will be considered as a baseline for the further alternative processes.

According to the proposed research methodology, the organization established in Veolia Eau to provide such planning processes was synthetized in Business Process Model Notation (BPMN). The main planning actors are the operations manager of each executing team as well as dedicated schedulers in charge of designing the intervention tours of a certain category of agents. These agents are those allocated to particular types of intervention, characterized by their high quantity and their small processing time, such as water meter reading interventions. Such intervention types generate a more combinatorial dimension to the tour definition. Depending on the 
intervention type, schedules proposed by dedicated schedulers are directly applied by agents or are reviewed by operations managers before their final application. This organization is depicted in Figure 3.

The dedicated schedulers also maintain a continuous watch for emergencies. When an emergency occurs, they are the first informed and have the responsibility of calling the appropriate agent and provide him with all the necessary information. Meanwhile each agent intervenes according to his planned schedule, until a hazardous event occurs: a call for emergency, the impossibility to execute an intervention... At the end of each intervention, weather it is correctly achieved or not, the agent reports the time and result into a tracking tool.

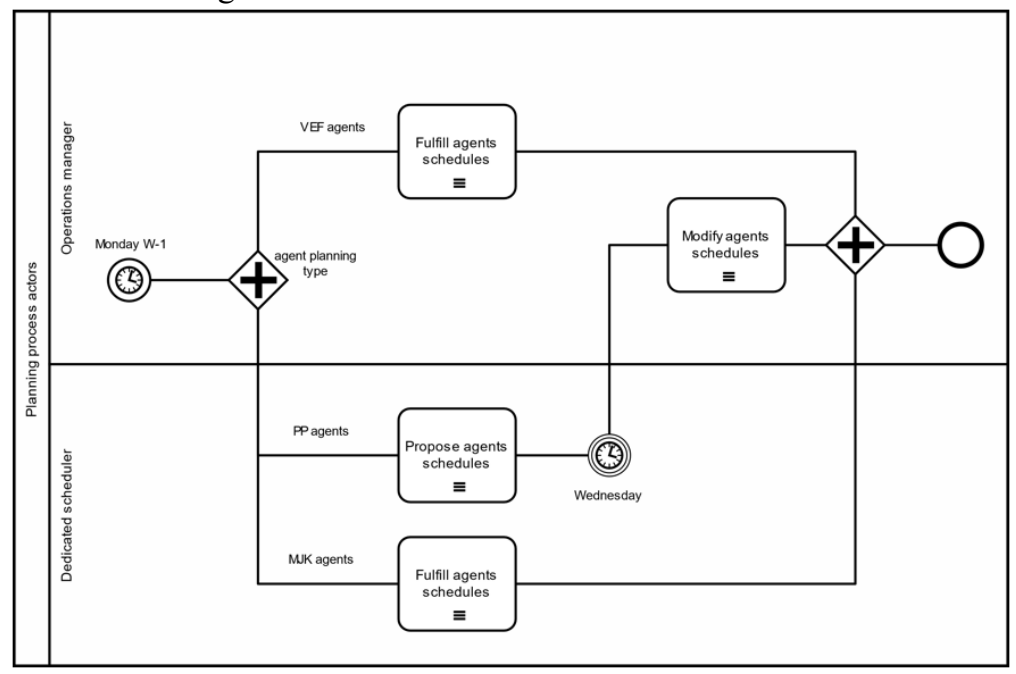

Figure 3. Scheduling organization in Veolia Eau

\section{Alternative Planning Processes Scenarios}

All the organizational constraints added to the general problem allow to find reasonable solutions to the planning problem manually, but they consequently narrow the field of planning solutions, and as a consequence probably diminish the quality of the adopted schedules. Relaxing such constraints in the planning problem requires the use of a computer tool embedding CVRP algorithms inspired from the literature integrating our specific additional constraints. To estimate the gain obtained by removing each constraint, one scenario will be built for each of them, namely (i) small geographic boundaries, (ii) pre-allocation of agents to specific types of interventions, (iii) planning horizon of one week. Figure 5 illustrates with basic fictional examples the potential limits induced by each of these constraints. In this figure, interventions are represented by circles and arrows of the same colour represent the intervention tour of an agent. In the first illustration, blue and orange areas represent two distinct geographic territories, for which the schedules are presently planned separately. Removing this separation could save transportation costs, especially for interventions located near the boundaries. In the second illustration, blue and orange circles are interventions of two different types, and we 
assume that the two agents in this figure have the skills to intervene on both of them. Again, transportation time can be saved by removing the constraint of one specific type of interventions for every agent. The dotted blue arrows in the last illustration is a tour executed the week after the tour represented in orange arrows, with the remaining interventions. It shows that anticipation over several weeks can degrade the quality of the first week schedules but improve the global planning in the long term.

The first alternative scenarios to develop are the relaxation of these three organizational constraints, while keeping the same comparative baseline presently used in Veolia EAU. The methodology presented in section 3 will allow us to compare objectively the potential gain obtained on realistic planning instances for each one of them. Keeping the same baseline used presently by Veolia Eau implies to work on planning strategies before the arrival of emergency interventions. The resilience of each scenario is still measurable by observing the final executed schedules after the simulation of emergency or delay occurrence during the realization of an intervention.
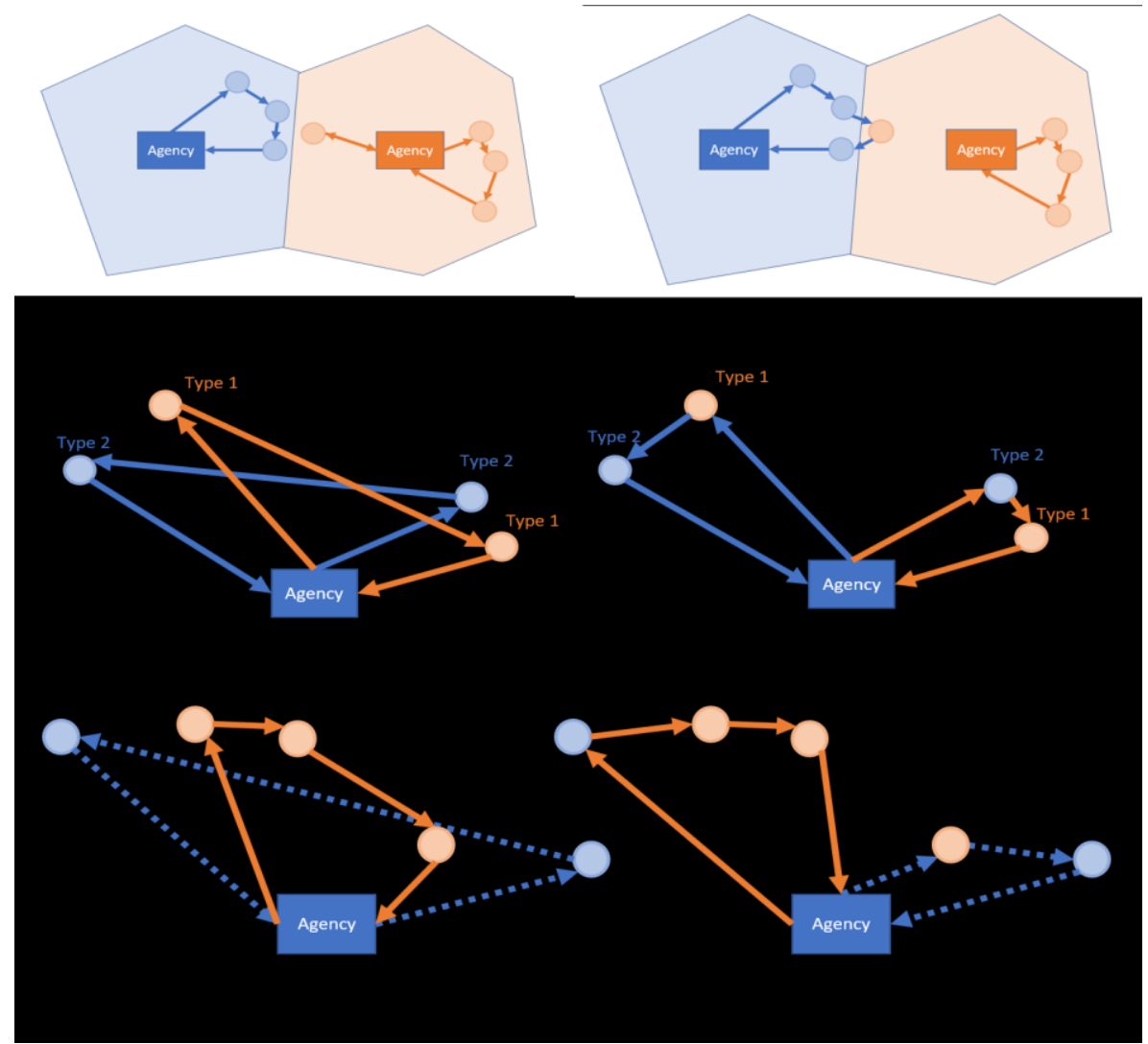

Figure 5. Limits induced by organisational constraints 


\section{Conclusion and Further Research}

The research project presented in this paper is in its infancy. If the real field planning water supply maintenance processes applied in Veolia Eau have already been described and modelled, the other steps described in the proposed research methodology remain to accomplish in order to provide answers to our core question: how to challenge and improve the maintenance planning strategies for water production and distribution network in an uncertain environment? The first planning alternative strategies presented in this study are tightly attached to the real field planning processes, in order to measure the cost (in terms of loss of efficiency and resilience) induced by changing a complex theoretical CVRP into a simplified and practical manually resolvable problem. However, other potential research avenues are considered with the integration of more sophisticated re-planning strategies, for a better consideration of the dynamic aspect of the problem.

\section{References}

[1] G. Capano, J.J Woo « Resilience and robustness in policy design: a critical appraisal », Policy Sciences, 2017, vol. 50, no 3, p. 399-426.

[2] S. Yamijala, S. D. Guikema, et K. Brumbelow, "Statistical models for the analysis of water distribution system pipe break data », Reliab. Eng. Syst. Saf., vol. 94, no 2, p. 282-293, févr. 2009.

[3] E. V. Ana and W. Bauwens, «Modeling the structural deterioration of urban drainage pipes: the state-of-the-art in statistical methods ", Urban Water J., vol. 7, nº 1, p. 47-59, févr. 2010

[4] G. B. Dantzig and J. H. Ramser, « The Truck Dispatching Problem », Manag. Sci., vol. 6, $\mathrm{n}^{\circ}$ 1, p. 80-91, oct. 1959.

[5] B. Kallehauge, J. Larsen, O. B. G. Madsen, and M. M. Solomon, «Vehicle Routing Problem with Time Windows », in Column Generation, G. Desaulniers, J. Desrosiers, et M. M. Solomon, Éd. Boston, MA: Springer US, 2005, p. 67-98.

[6] C. Archetti, M. G. Speranza, and D. Vigo, «Chapter 10: Vehicle Routing Problems with Profits », in Vehicle Routing, 0 vol., Society for Industrial and Applied Mathematics, 2014, p. 273-297.

[7] A. Subramanian, E. Uchoa, A. A. Pessoa, and L. S. Ochi, «Branch-and-cut with lazy separation for the vehicle routing problem with simultaneous pickup and delivery », Oper. Res. Lett., vol. 39, nº 5, p. 338-341, sept. 2011.

[8] J. C. Beck, P. Prosser, and E. Selensky, "Vehicle Routing and Job Shop Scheduling: What's the difference? ", in Proceedings of the Thirteenth International Conference on Automated Planning and Scheduling, 2003, p.267-276.

[9] P. Toth et D. Vigo, Éd., The vehicle routing problem. Philadelphia: Society for Industrial and Applied Mathematics, 2002.

[10] R. Baldacci, A. Mingozzi, and R. Roberti, «Recent exact algorithms for solving the vehicle routing problem under capacity and time window constraints », Eur. J. Oper. Res., vol. 218, $\mathrm{n}^{\mathrm{o}}$ 1, p. 1-6, avr. 2012.

[11] V. Pillac, M. Gendreau, C. Guéret, and A. L. Medaglia, «A review of dynamic vehicle routing problems », Eur. J. Oper. Res., vol. 225, n 1, p. 1-11, 2013. 
[12] A. Clement, D. Kamissoko, F. Marmier, and D. Gourc, «The robustness of the round planning face to risks of road freight transport », in 2018 4th International Conference on Logistics Operations Management (GOL), avr. 2018, p. 1-7.

[13] R. Bent and P. Van Hentenryck, "Online Stochastic and Robust Optimization », in Advances in Computer Science - ASIAN 2004. Higher-Level Decision Making, Berlin, Heidelberg, 2005, p. 286-300.

[14] W. Kool, H. van Hoof, J. Gromicho, and M. Welling, «Deep Policy Dynamic Programming for Vehicle Routing Problems », ArXiv Cs Stat, 2021.

[15] A. A. Juan, J. Faulin, S. E. Grasman, M. Rabe, and G. Figueira, "A review of simheuristics: Extending metaheuristics to deal with stochastic combinatorial optimization problems », Oper. Res. Perspect., vol. 2, p. 62-72, déc. 2015.

[16] M. Abdirad, K. Krishnan, and D. Gupta, "A two-stage metaheuristic algorithm for the dynamic vehicle routing problem in Industry 4.0 approach », J. Manag. Anal., vol. 8, $\mathrm{n}^{\circ}$ 1, p. 69-83, janv. 202.

[17] M. Abdirad, K. Krishnan, and D. Gupta, « A Three-Stage Algorithm for the Large Scale Dynamic Vehicle Routing Problem with an Industry 4.0 Approach », ArXiv200811719 Cs Math, oct. 2020.

[18] A. Chibani, X. Delorme, A. Dolgui, and H. Pierreval, «Dynamic optimisation for highly agile supply chains in e-procurement context », Int. J. Prod. Res., vol. 56, $\mathrm{n}^{\circ}$ 17, p. 5904-5929, sept. 2018.

[19] Q. Schoen, M. Lauras, S. Truptil, F. Fontanili, and A.-G. Anquetil, "Towards a Hyperconnected Transportation Management System: Application to Blood Logistics ", in Collaboration in a Hyperconnected World, Cham, 2016.

[20] H. Lei, G. Laporte, and B. Guo, « A generalized variable neighborhood search heuristic for the capacitated vehicle routing problem with stochastic service times », TOP, vol. 20, $\mathrm{n}^{\mathrm{o}}$ 1, p. 99-118, avr. 2012.

[21] J.-Q. Li, P. B. Mirchandani, and D. Borenstein, «The vehicle rescheduling problem: Model and algorithms », Networks, vol. 50, nº 3, p. 211-229, 2007.

[22] A. M. Law, A. M. Law, W. D. A. KELTON, W. D. Kelton, and D. M. Kelton, Simulation Modeling and Analysis. McGraw-Hill, 2000.

[23] M. L. Patten and M. Newhart, Understanding Research Methods: An Overview of the Essentials. Taylor \& Francis, 2017.

[24] Z. Shi, E. Jeannot, and J. J. Dongarra, «Robust task scheduling in non-deterministic heterogeneous computing systems ", in 2006 IEEE International Conference on Cluster Computing, sept. 2006, p. 1-10.

[25] L.-C. Canon and E. Jeannot, « A Comparison of robustness metrics for scheduling DAGs on heterogeneous systems ", in 2007 IEEE International Conference on Cluster Computing, sept. 2007, p. 558-567.

[26] L.-C. Canon and E. Jeannot, «Evaluation and Optimization of the Robustness of DAG Schedules in Heterogeneous Environments », IEEE Trans. Parallel Distrib. Syst., vol. 21, no 4 , p. 532-546, avr. 2010. 